\title{
Role of excited atoms in Lidar measurements of the middle and upper atmosphere
}

\author{
Vasily Bychkov ${ }^{1, \star}$, Andrey Perezhogin ${ }^{1, \star \star}$ and I'ya Seredkin ${ }^{1}$ \\ ${ }^{1}$ Institute of Cosmophysical Research and Radio Wave Propagation FEB RAS (IKIR FEB RAS), \\ 684034, Mirnaya str., 7, Paratunka, Elizovskiy district, Kamchatka, Russia
}

\begin{abstract}
The paper presents the results of a lidar study of the atmosphere in the altitude region of 25-400 km, obtained at the lidar station of Kamchatka in 2008-2016. The development of methods for lidar measurements and signal processing is discussed. The method that takes into account the aftereffects of photoelectric multipliers and the technique to measure the background signal is described. These tools allow the authors to include the mesosphere and the upper atmosphere into the altitude region investigated by the lidar method. The results of the investigation of the backscattering lidar signal from the altitude region of $100-400 \mathrm{~km}$ caused by the resonance scattering on exited components of upper atmosphere are discussed.

Keywords: atmosphere, lidar, stratosphere, mesosphere, scattering, sounding
\end{abstract}

\section{Introduction}

Increased backscattering at the wavelength of $532 \mathrm{~nm}$ in the region of the upper atmosphere and its correlation with the critical frequency of ionospheric F2 layer on some days was detected during the investigation of the aftereffect of photomultiplier (PMT) Hamamatsu H8259-01 applied in the lidar in Kamchatka. Resonance behavior of a backscattering signal was discovered in the region of $100-400 \mathrm{~km}$. The backscattering signal in the region of $150-300 \mathrm{~km}$ was investigated in the papers [1-3]. According to the data of ionosphere and lidar observations at the wavelength of $532 \mathrm{~nm}$ it was shown that during soft $(0.1-10 \mathrm{keV})$ electron precipitations into the atmosphere, the total lidar signal from the region of heights of 100-400 km may correlate with plasma content in the region of maximum of nighttime ionosphere F2 layer. Analysis of the geophysical state, which accompanied the phenomenon, allowed to make the conclusion that the possible physical mechanism explaining these correlations is the resonance scattering at exited ions of nitrogen atom forming during soft (0.1-10 $\mathrm{keV}$ ) electron precipitations into the ionosphere.

The content of nitrogen ions at ionospheric heights is units of percentage by day and negligibly small by night. The papers $[4,5]$ present the results of registration of the resonance scattering at exited oxygen ions, which is the main charged component in the ionospheric F2 layer. The wavelength of the sounding emission of $561.107 \mathrm{~nm}$ was chosen for the study of resonant scattering by atomic oxygen ions, as the most appropriate for the equipment.

\footnotetext{
^ e-mail: vasily.v.bychkov@gmail.com

$\star \star$ e-mail: d72156@gmail.com
} 


\section{Equipment}

In the lidar observations of 2015-2016 a dual-frequency lidar with a laser Brilliant-B was used to generate $532.08 \mathrm{~nm}$ radiation and a dye laser TDL-90 with an pumping laser YG982E to generate radiation with the wavelength of more than $532.08 \mathrm{~nm}$. For example, we describe the scheme for the wavelength of $561.107 \mathrm{~nm}$. Dye Rhodamine 590 was used. The both lasers had the frequency of 10 Hz. Pulse energy at the wavelength of $532.08 \mathrm{~nm}$ was $\sim 400 \mathrm{~mJ}$ and about $150 \mathrm{~mJ}$ at the wavelength of $561.107 \mathrm{~nm}$. The receiving telescope has a parabolic mirror with a diameter of $60 \mathrm{sm}$. The angle of view of the receiver was chosen equal $0.2 \mathrm{mrad}$.

The scheme of possible dipole transitions falling within the radiation band of TDL-90 and Brilliant - B is shown in Table 1, where OII is $O^{+}$ion, NII is $N^{+}$ion, NIII is $N^{++}$ion. It is taken into account that based on the ratings, the radiation spectrum widths at the half maximum are $0.04 \mathrm{~nm}$ for the laser Brilliant-B and $0.005 \mathrm{~nm}$ for laser TDL.

Table 1. Dipole transitions of excited ions of oxygen and nitrogen atoms falling within the radiation bands of lasers TDL-90 with Rh-590 dye and Brilliant-B [6].

\begin{tabular}{|l|l|l|l|l|l|l|l|l|l|}
\hline & $\begin{array}{l}\text { Compo- } \\
\text { nent }\end{array}$ & $\begin{array}{l}\text { Wavelength } \\
\text { Air }(\mathrm{nm})\end{array}$ & $\mathrm{A}_{k i}\left(\mathrm{~s}^{-1}\right)$ & Lower Level & Term & $\mathrm{J}$ & $\begin{array}{l}\text { Upper } \\
\text { Level }\end{array}$ & Term & $\mathrm{J}$ \\
\hline 1 & OII & 561.1072 & $2,14 \mathrm{e}+06$ & $2 s^{2} 2 p^{2}\left({ }^{1} \mathrm{~S}\right) 3 s$ & ${ }^{2} \mathrm{~S}$ & $1 / 2$ & $2 s^{2} 2 p^{2}\left({ }^{3} \mathrm{P}\right) 4 \mathrm{p}$ & ${ }^{2} \mathrm{P}^{\circ}$ & $1 / 2$ \\
\hline 2 & NIII & 532.0870 & $5.68 \mathrm{e}+07$ & $2 \mathrm{~s} 2 \mathrm{p}\left({ }^{3} \mathrm{P}^{\circ}\right) 3 \mathrm{p}$ & ${ }^{2} \mathrm{D}$ & $5 / 2$ & $2 \mathrm{~s} 2 \mathrm{p}\left({ }^{3} \mathrm{P}^{\circ}\right) 3 \mathrm{~d}$ & ${ }^{2} \mathrm{~F}^{\circ}$ & $7 / 2$ \\
\hline 3 & NII & 532.0958 & $2.52 \mathrm{e}+07$ & $2 \mathrm{~s} 2 \mathrm{p}^{2}\left({ }^{4} \mathrm{P}\right) 3 \mathrm{p}$ & ${ }^{5} \mathrm{P}^{\circ}$ & 1 & $2 \mathrm{~s} 2 \mathrm{p}^{2}\left({ }^{4} \mathrm{P}\right) 3 \mathrm{~d}$ & ${ }^{5} \mathrm{P}$ & 2 \\
\hline
\end{tabular}

The signal received by the telescope is sent to a light guide as a parallel beam through the lenses of a receiving unit. A spectrum dividing plate is set in the light guide at the angle of $45 \circ$ degrees to the direction of beam propagation. Radiation which has more than $532 \mathrm{~nm}$ wavelength is omitted by the plate. Radiation with the wavelength of $532 \mathrm{~nm}$ and less is reflected at the angle of $90^{\circ}$ into another light guide. Then, the separated radiation fluxes are sent to two PMT Hamamatsu H8259-01, connected to photon counters Hamamatsu M8784. The duration of the counter gate is $10 \mu \mathrm{s}$ which corresponds to the layer of $1.5 \mathrm{~km}$ taking into the account the beam two-way propagation.

Control of ionosphere state was carried out according to the data of "Parus" ionosonde which replaced the Automatic Ionospheric Station (AIS) in August 2015.

\section{Measurement method and signal processing}

In the investigation of aerosol distribution in the middle atmosphere, the scattering ratio was taken as the main parameter. It was determined as $\mathrm{R}=\left(\beta_{a}+\beta_{m}\right) / \beta_{m}=1+\beta_{a} / \beta_{m}$, here $\beta \mathrm{a}, \beta \mathrm{m}$ are the coefficients of back aerosol and molecular scattering. The dynamic range of back scattering signals is very large and cannot be provided by a one PMT. The near zone (from 0 to $20 \mathrm{~km}$ ) signals were excluded by electronically PMT locking. Nevertheless, $\mathrm{R}(\mathrm{H})$ profile recovered only to the heights of $50-60 \mathrm{~km}$. Then the scattering ratio rapidly increases caused by aftereffect pulses. The results of investigation of photomultiplier tube (PMT) aftereffect were published in the paper [7]. It was shown that in the region of $60-90 \mathrm{~km}$, the aftereffect pulses may be considered by detection of an exponent in a signal from the region of $\sim 100-150 \mathrm{~km}$ by least square method and its subtraction from the basic signal. Application of this method allows us to recover the profile of the relation of scattering $\mathrm{R}=\left(\beta_{a}+\beta_{m}\right) / \beta_{m}$ from the heights of $\sim 50-60 \mathrm{~km}$ to $75-90 \mathrm{~km}$. Thus, the whole region of the middle atmosphere $(25-80 \mathrm{~km})$ becomes available for investigation by lidar sounding method. 
We should mention the technique to measure the background signal which was suggested within the course of investigation of PMT aftereffect. Analysis of a night summed signal showed that the signal at altitudes of 150-600 km behaves differently on different days. Usually on the graph, it is a straight line parallel to the height axis. However, on some days the signals decreases after $100 \mathrm{~km}$ and remains inclined to the height axes up to 500-600 km. Examples of such signals, obtained on March 28 and September 14, 2008, are shown in Fig. 1. The technical settings of the lidar were the same on the both days. The time of signal accumulation was about the same ( $\sim .5$ hours). The signal value differences at the height of $600 \mathrm{~km}$ are explained by full Moon during the observations on September 14 and its absence on March 28, 2008. The difference in signal behavior in the region of 100-500 $\mathrm{km}$ cannot be explained by the aftereffect. Moon illumination on September 14 created additional background. The signal total level increased and PMT aftereffects had to manifest brighter.

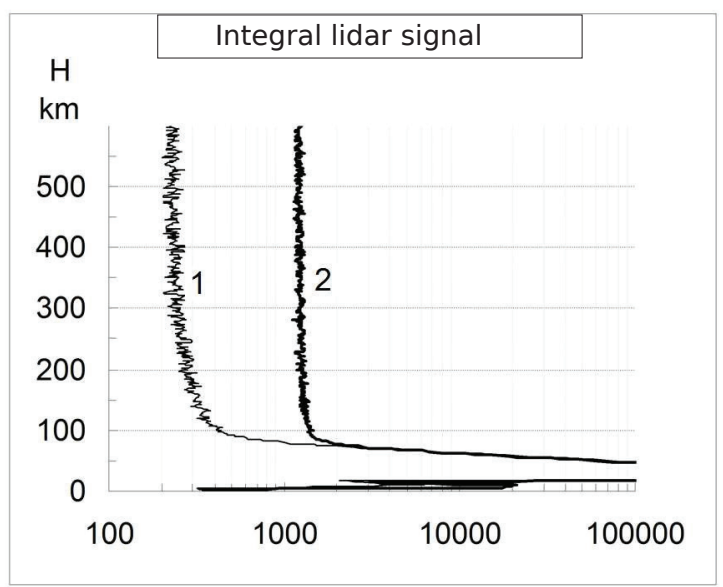

Figure 1. Total lidar signal obtained on March 28, 2008 (1) and September 14, 2008 (2).

It was concluded that background measurements within the interval of 2-4 ms (150-600 km) after a laser pulse sending may contain some additional signals. For this reason the background signal was measured as the mean value measured from the 20th to 24th ms with a step of $10 \mu s$ after each laser pulse sending. The background signal measured in such a way does not contain aftereffect pulses and is provided by good data collection.

In the course of investigation of PMT aftereffect during the sounding at the wavelength of 532 $\mathrm{nm}$, an unusual burst of the total lidar signal from the region of $200-400 \mathrm{~km}$ was detected. Further studies have shown that the increase in the lidar signal from the heights of the upper atmosphere is explained by resonance scattering on excited atomic nitrogen atoms caused by super thermal electron precipitation (0.1-10 keV) into the ionosphere [1-3]. Fig. 2 illustrates the results of ionospheric and lidar sounding which were discussed in these papers. To show the importance of proper treatment of the background signal during the measurements in the upper atmosphere, the figures were somewhat modified.

All the curves of Fig. 2a, 2c were plotted according to the lidar data with 15-minute accumulation time (9000 laser pulses). They are a total signal from the layers of $100 \mathrm{~km}$ thick. The curves in Fig. $2 \mathrm{a}, 2 \mathrm{c}$ are plotted based on the lidar data applying background values measured at 20-24 ms after each laser pulse sending. When plotting the curves for Fig. 3a, 3c, we used the background value as an average over 100 strobes $(150 \mathrm{~km})$ in the region of $350-500 \mathrm{~km}$. 

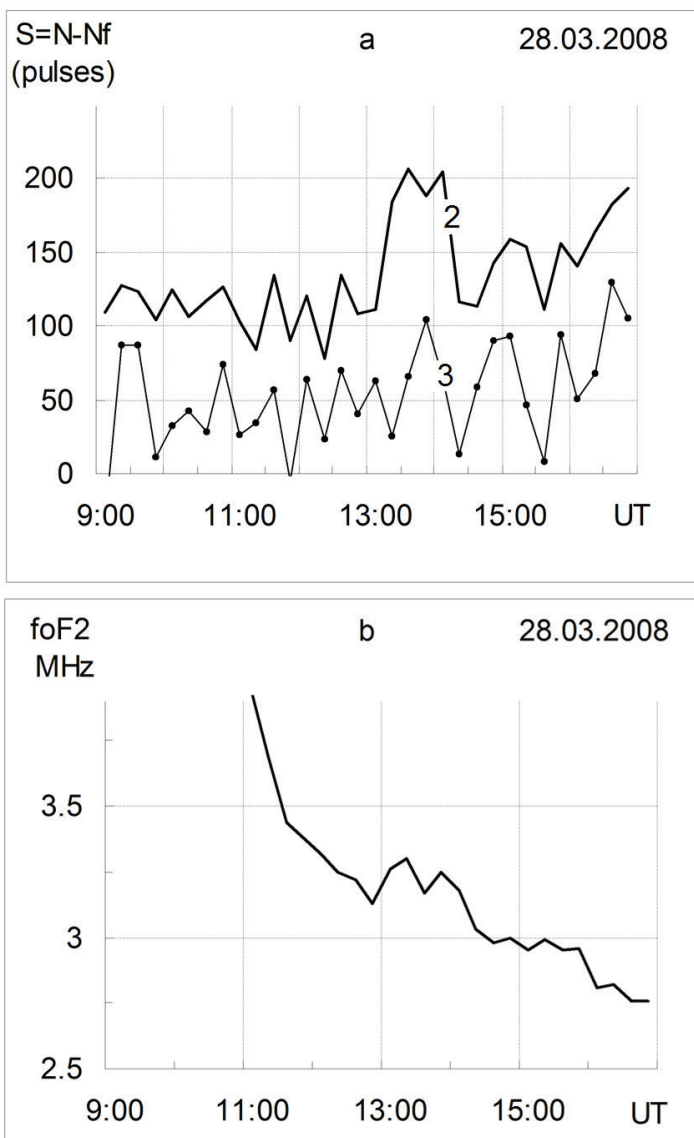
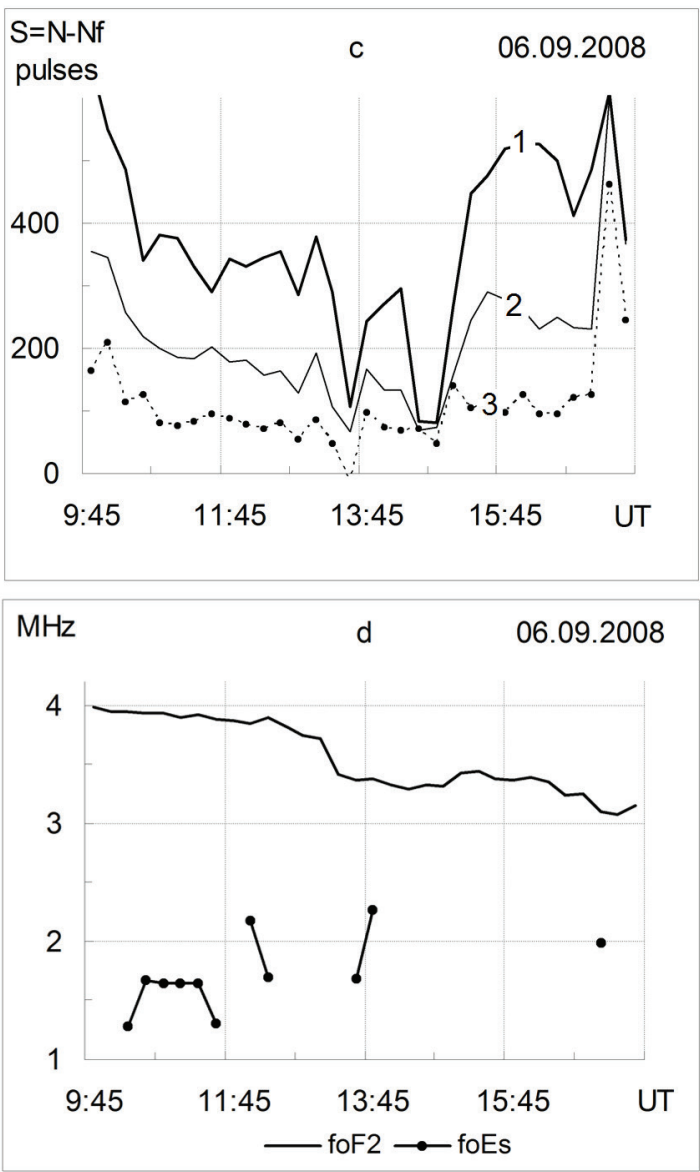

Figure 2. Total lidar "signal-background" (a, c) from the regions of 100-200 km (curve 1) and 200-300 km (curves 2, 3) and foF2 graph (b, d) during lidar observations on March 28 and September 6, 2008.

The curves $2 \mathrm{a}, 2 \mathrm{c}$ have synchronous changes with foF2 that is explained by the resonance scattering at excited ions of atomic nitrogen. The dependence of curve $3 \mathrm{a}$ and $3 \mathrm{c}$ forms on the choice of background averaging interval in the region of 300-600 km and signal decrease for such a choice of the background values allow us to make the conclusion that the background signal measured before the beginning of $5 \mathrm{~ms}$ after laser pulse sending cannot be used to investigate the upper atmosphere. In the middle atmosphere, estimating the background signal by its average value over the upper strobe can lead to unpredictably underestimated results. For example, to values of the scattering ratio $\mathrm{R}$ smaller than 1.

\section{Some results in the upper atmosphere}

The papers $[4,5]$ present Nh-profiles of atomic oxygen ion excited states obtained in the result of two-frequency sounding at the wavelength of 532.08 and $561.107 \mathrm{~nm}$. The frequency of 561.107 $\mathrm{nm}$ corresponds to dipole transition between excited states of atomic oxygen long-lived ion. Fig. 3 illustrates the Nh-profiles of atomic nitrogen excited states obtained from the same initial data as for 
Fig. 2. As long as the resonance scattering signal at atomic nitrogen ions is several times less than the scattering signal at oxygen ions, the profiles include only the time interval which corresponds to the maximum value of resonance scattering signal shown in Fig. 2. The time of signal accumulation is $75 \mathrm{~min}$ and is marked in the both figures.

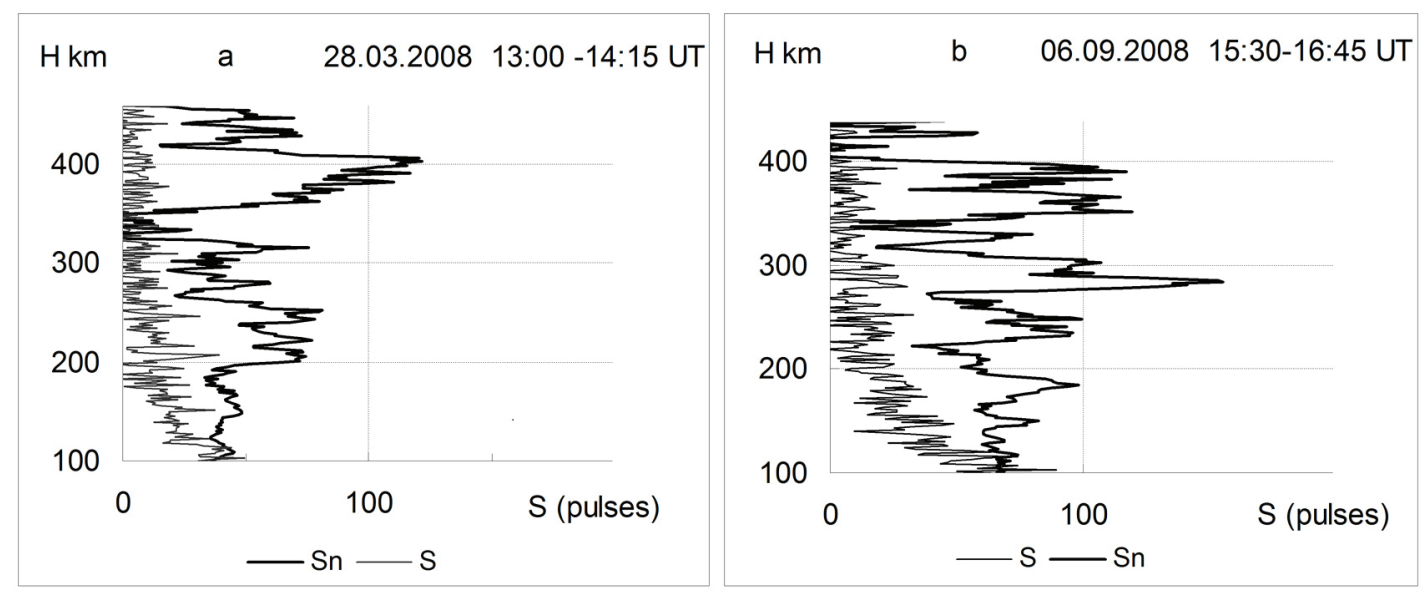

Figure 3. Total lidar signal S and normalized signal Sn on March 28 and September 6, 2008.

Signal $\mathrm{S}$ is the backscattering total signal accumulated over the 75 minutes except the measured background. Sn signal is the $\mathrm{S}$ signal normalized to a geometric factor $\left(\mathrm{H}^{2} / 100^{2}\right)$ relatively its value at the height of $100 \mathrm{~km}$. Sn data are smoothed by the moving average method with the window of $10.5 \mathrm{~km}$. In fact, these are curves of the recorded content of nitrogen atom ion excited states. Figure 3 a contains two separate areas of increased scattering, which may be the basis for estimating the characteristic energy of the spectra of precipitated electrons. A sharp decrease in the signal Fig. 3a at altitudes of 200 and $\sim 350 \mathrm{~km}$ is characteristic of the rate of ionization caused by monoenergetic electron fluxes. Electron precipitations may occur within very short time periods. Decrease of accumulation time should result in the detection of such defined areas that requires recorded signal general level increase.

\section{Conclusions for mesospheric observations}

Resonance scattering on excited components of the upper atmosphere should also be manifested in the mesosphere during relativistic electron precipitation. The paper [Bychkov et al, 2012] illustrates the data on the correlation between the ratio of scattering $\mathrm{R}=\left(\beta_{a}+\beta_{m}\right) / \beta_{m}$ in layers of $\sim 5 \mathrm{~km}$ thick and ionospheric parameter $\mathrm{f}_{\min }$. Explanations of this correlation have not been proposed. The total coefficient of scattering was determined according to the results of lidar observations at the wavelength of $532 \mathrm{~nm}$.

Analysis of ionospheric and lidar data on January 18 and 23, February 18 and 19, 2008 discovered correlations between the values of ionospheric parameter $f_{\min }$ and the total ratio of scattering $\mathrm{R}$ in layers of $\sim 5 \mathrm{~km}$ thick in the mesospheric region. Correlation coefficient values were $0.68,0.77,0.5$ and 0.5 , respectively. When calculating the molecular scattering coefficient, we used temperature data measured on Aura satellite.

On all the mentioned days according to Demeter satellite flying westward and eastward to Kamchatka during the lidar observations, significant fluxes of relativistic electrons with the energies in 
range 90-526 keV were recorded when the satellite crosses the latitude of the lidar station of Kamchatka $(53 \mathrm{~N})$. Ionization rate in the mesosphere region caused by the precipitation of these electrons was calculated from the spectrum of electrons measured on Demeter satellite on January 18, 2008. The obtained ionization rate exceeds the values generally accepted in modeling of D layer of ionosphere by more than one order.
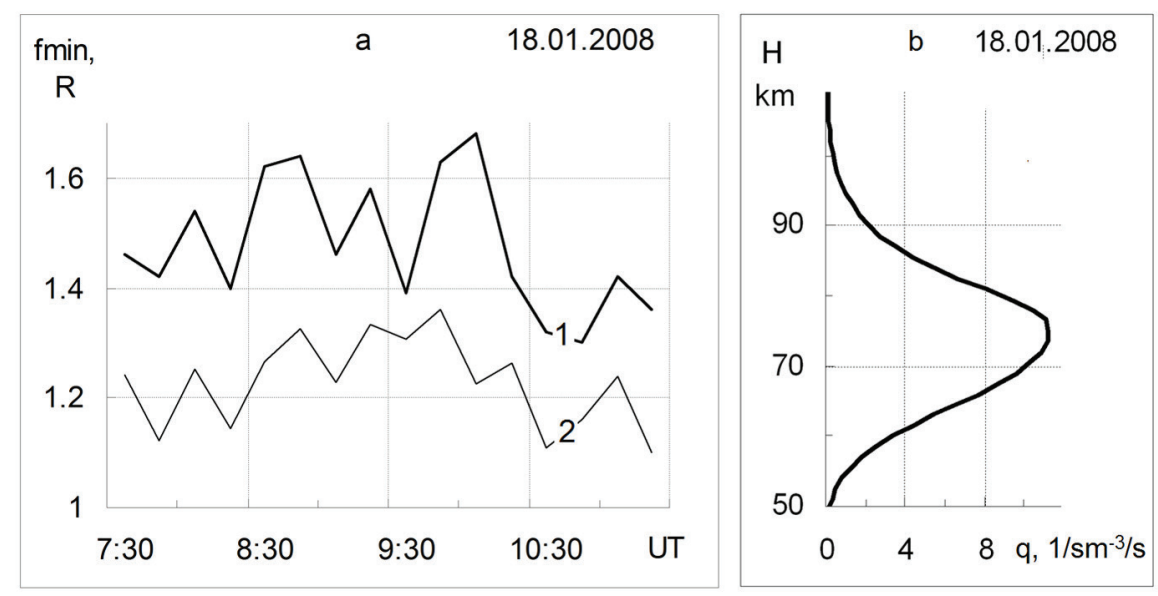

Figure 4. Values of $\mathrm{f}_{\min }(4 \mathrm{a}, 1)$ and of lidar signal $(4 \mathrm{a}, 2)$ from the region of 64.5-70.5 km on January 18, 2008 (a), and ionization rate caused by precipitated electrons measured on Demeter at 10:10 UT (b) [8].

As an example, Fig. 4 illustrates the data from the paper [8]. Fig. 4 shows $f_{\min }$ values and the average relation of scattering coefficients $\mathrm{R}$ in the region of $64.5-70.5 \mathrm{~km}$. Correlation coefficient between the curves is 0.68 . Fig. $4 \mathrm{~b}$ represents the ionization rate caused by relativistic electrons calculated according to the data from Demeter satellite. The calculations were made according to the spectrum of precipitated electrons measured on the satellite Demeter by the method described in the paper [9].

In the same paper [8] the authors estimate the possibility of water vapor condensation in the mesosphere. Water vapor pressure over ice necessary for the condensation to begin was calculated by Magnus' formula. To estimate water content in the mesosphere, the measurement data on Aura satellite were used. It was obtained that in winter time during average temperature conditions in the mesosphere, the water content is two orders less than that necessary for condensation and for aerosol formation. Aerosol formations were observed in the mesosphere on January 18, 2008 and on other days in the region of heights of $60-80 \mathrm{~km}$.

The results of the papers [1-3] allow us to suggest the mechanism of formation of additional lidar signals in the mesosphere. Changes of $f_{\min }$ are associated with the change of free electron content in the upper layers of ionospheric $\mathrm{D}$ region. Increase of $f_{\min }$ means the increase of absorption of incident radio wave low frequencies. In the absence of collisions of free electrons with neutral particles, an electromagnetic wave oscillates a free electron. A moving electron radiates at the same frequency and absorption does not occur. A radio wave loses its energy during collisions of a vibrating electron with a neutral particle. Absorption coefficient per a path unit $\mathrm{K} \sim \mathrm{N} / v$, where $\mathrm{N}$ is free electron content, $v$ is the frequency of collisions of electrons with neutrals. In the Earth atmosphere, absorption of radio waves with the frequency of units of $\mathrm{MHz}$ mainly occurs in the region of about $70 \mathrm{~km}$ where absorption coefficient is maximal. The content of free electrons decreases at the height of less than $70 \mathrm{~km}$. At higher altitudes the frequency of collisions of electrons with neutrals drops with content 
of neutrals decreasing. Thus, increase of $\mathrm{f}_{\text {min }}$ indicates the growth of electron content which means ionization rate increase in nighttime conditions.

The main component of the atmosphere at these heights is molecular nitrogen. Ionization by energetic electrons is accompanied by photochemical reactions, for example, by the processes of dissociation of molecular nitrogen, nitrogen oxide and many other reactions causing the formation of excited atoms and ions of atomic nitrogen. The conditions for participation of these ions in the process of resonance scattering at the heights of $70 \mathrm{~km}$ do not differ from the conditions of the upper atmosphere though the frequency of ion-neutral collisions at the heights of $\sim 70 \mathrm{~km}$ increases. The frequency of electron-neutral collisions may be calculated as:

$$
v[N]=0.81 \cdot 10^{-10}(T / M)^{1 / 2} \cdot N\left(c^{-1}\right)
$$

where $\mathrm{T}$ is temperature, $\mathrm{M}$ - molecular weight, $\mathrm{N}$ - particle concentration in the atmosphere [10] . Substituting $\mathrm{N}$ equal to $10^{15} \mathrm{sm}^{-3}$ for the height of $70 \mathrm{~km}$, we obtain $v \sim 5 \cdot 10^{5} \mathrm{~s}^{-1}$, i.e. $500 \mathrm{kHz}$. Taking into account that the frequency of ion-neutral collisions is at least two orders less than that of electrons, it becomes clear that for excited ions with radiative lifetime of less than $10^{-6} \mathrm{~s}$, the increase of collisions at the heights of $70 \mathrm{~km}$ does not play significant role.

Thus, the lidar signal increase from mesospheric heights during relativistic electron precipitations may be explained by the appearance of excited ions of atomic nitrogen and the conditions for resonance scattering at the wavelength of $532 \mathrm{~nm}$. This suggestion explains the correlation of the lidar signal in the region of heights of $70 \mathrm{~km}$ with ionospheric parameter $\mathrm{f}_{\min }$ observed in 2008 .

\section{Conclusions}

Analysis of the obtained results allows us to make the following conclusion:

- it is shown that a correct account of the background signal during lidar studies of the upper and middle atmosphere can be of fundamental importance;

- when analyzing the results of mesospheric sounding, we should take into the account the frequencies corresponding to dipole transitions between excited states of atmospheric main components in laser radiation spectrum. Precipitations of relativistic electrons may cause additional lidar signal and formation of imaginary aerosol formations in the mesosphere;

- lidar investigations of the upper atmosphere may provide the data on the spectra of precipitated electrons with the energies of $0.1-10 \mathrm{keV}$;

- the main features of resonance scattering manifestation may be the inclination to the height axis of lidar total nighttime signal at the heights of more than 100-150 km and the correlation of total signals from different regions of the upper atmosphere between each other. Ionospheric data may serve as aditional source of information.

The paper was supported by FEB RAS Grant No. 12-I-OFN-16, RFBR Grant No. 16-05-00901a, RSCF No. 1411-00194.

\section{References}

[1] V. Bychkov, Y.A. Nepomnyashchii, A. Perezhogin, B. Shevtsov, N. Polekh, Atmospheric and Oceanic Optics 27, 297 (2014)

[2] V. Bychkov, Y.F. Nepomnyashchii, A. Perezhogin, B. Shevtsov, Atmospheric and Oceanic Optics 28, 303 (2015) 
[3] V.V. Bychkov, Y.A. Nepomnyashchiy, A.S. Perezhogin, B.M. Shevtsov, Resonance scattering at excited atoms and ions of the upper atmosphere as a possible mechanism for ionosphere investigations, in XXI International Symposium Atmospheric and Ocean Optics. Atmospheric Physics (International Society for Optics and Photonics, 2015), pp. 968073-968073

[4] V.V. Bychkov, A.S. Perezhogin, I.N. Seredkin, B.M. Shevtsov, Lidar investigations of the scattering of the upper and middle atmosphere, in XXII International Symposium Atmospheric and Ocean Optics. Atmospheric Physics (International Society for Optics and Photonics, 2016), pp. $100355 \mathrm{R}-100355 \mathrm{R}$

[5] V.V. Bychkov, A.S. Perezhogin, I.N. Seredkin, B.M. Shevtsov, V.N. Marichev, Atmospheric and Oceanic Optics 30, 209 (2017)

[6] Nist atomic spectra database lines form, https://physics.nist.gov/PhysRefData/ASD/ lines_form.html

[7] V. Bychkov, A. Perezhogin, B. Shevtsov, V. Marichev, P. Novikov, A. Cheremisin, Optika Atmos. Okeana 24, 107 (2011)

[8] V. Bychkov, A. Perezhogin, B. Shevtsov, V. Marichev, G. Matvienko, A. Belov, A. Cheremisin, Atmospheric and Oceanic Optics 25, 228 (2012)

[9] M. Demin, M.: Fizmatlit 2, 92 (2008)

[10] N. Shefov, A. Semenov, V.Y. Khomich, Geos, Moscow (2006) 